\title{
Expression of a novel oncofetal mRNA-binding protein IMP3 in endometrial carcinomas: diagnostic significance and clinicopathologic correlations
}

Cuizhen $\mathrm{Li}^{1}$, Victor Zota ${ }^{2}$, Bruce A Woda ${ }^{2}$, Kenneth L Rock ${ }^{2}$, Armando E Fraire ${ }^{2}$, Zhong Jiang ${ }^{2}$, Di Lu ${ }^{2}$, Bo Xu ${ }^{2}$, Karen Dresser ${ }^{2}$, Christopher V Lutman ${ }^{1}$ and Andrew H Fischer ${ }^{2}$

${ }^{1}$ Riverside Methodist Hospital, Columbus, OH, USA and ${ }^{2}$ Department of Pathology, University of Massachusetts Medical School, Worcester, MA, USA

\begin{abstract}
Insulin-like growth factor-II mRNA-binding protein 3 (IMP3) is a newly identified oncofetal mRNA-binding protein that is involved in embryogenesis and carcinogenesis of some malignant neoplasms. To investigate the diagnostic and clinicopathologic significance of this protein in endometrial carcinomas, we evaluated immunohistochemical expression of IMP3 in the two most common forms of endometrial malignancies, endometrioid adenocarcinoma and serous carcinoma. We selected 167 endometrial adenocarcinoma cases including 122 cases of endometrioid adenocarcinoma and 45 cases of serous carcinoma. Twenty samples of benign endometrium obtained from 20 patients with nonmalignant uterine lesions were used as controls. Positive immunohistochemical stain for IMP3 was identified in all serous carcinoma cases, among which, $39(86 \%)$ and $3(7 \%)$ cases showed IMP3 immunoreactivity in $>50 \%$, and $21-50$, or $6-20 \%$ of tumor cells, respectively. Immunohistochemical reaction intensity for IMP3 was identified to be strong in $38(84 \%)$ and intermediate in $7(16 \%)$ cases of serous carcinoma. Fifty-four $(44 \%)$ cases of endometrioid adenocarcinoma were negative for IMP3. Thirty (25\%), $20(16 \%), 10(8 \%)$, and $8(7 \%)$ cases of endometrioid adenocarcinoma demonstrated positive immunoreactivity for IMP3 in 1-5, 6-20, 21-50, and $>50 \%$ of the tumor cells. Strong IMP3-staining intensity was noted in $34(28 \%)$, intermediate in $26(21 \%)$, and weak in $8(7 \%)$ cases of endometrioid adenocarcinoma. All 20 control cases were negative for IMP3. To compare p53 with IMP3 expressions, we found that $35(78 \%)$ of the serous carcinoma cases showed strong p53 immunohistochemical activity in $>50 \%$ of the tumor cell nuclei. In contrast, 11 of $112(10 \%)$ endometrioid adenocarcinoma cases demonstrated strong p53 positivity in $>50 \%$ of the tumor cell nuclei. In conclusion, our findings demonstrate significant expression of IMP3 in serous carcinoma as compared to endometrioid adenocarcinoma $(\boldsymbol{P}<0.0001)$. Expression of IMP3 and p53 may be helpful biomarkers in the distinction of endometrial serous carcinoma from endometrioid adenocarcinoma. In addition, expression of IMP3 in endometrioid adenocarcinoma correlates with higher nuclear and architecture grades of the tumor $(P=0.0000$ and $P=0.0002$, respectively).

Modern Pathology (2007) 20, 1263-1268; doi:10.1038/modpathol.3800960; published online 21 September 2007
\end{abstract}

Keywords: IMP3; endometrioid adenocarcinoma; serous carcinoma

Carcinomas of the endometrium are the most common malignancy of the gynecologic tract and rank fourth in incidence among invasive tumors in women, following breast, lung, and colon cancers. Endometrial carcinomas exhibit a broad morphologic spectrum thought to be the consequence of variable cellular differentiation. The most fre-

Correspondence: Dr C Li, MD, PhD, Riverside Methodist Hospital, 3535 Olentangy River Road, Columbus, OH 43214, USA.

E-mail: cuizhenli@yahoo.com

Received 19 March 2007; revised 18 July 2007; accepted 31 July 2007; published online 21 September 2007 quent subtype of endometrial carcinoma is endometrioid adenocarcinoma ( $>80 \%$ ), followed by serous carcinoma $(10 \%)$. Endometrioid adenocarcinoma that is confined to the uterus has a very favorable outcome with a 5-year-survival rate of $87.4 \%$ reported in the Annual Report (AR) of FIGO (International Federation of Gynecology and Obstetrics). ${ }^{1}$ In contrast, serous carcinoma is infrequent compared with endometrioid adenocarcinoma, and has a much higher propensity for spreading beyond the uterus early in the disease process, resulting in a much poorer clinical outcome. Therefore, it is very important for proper clinical management to 
distinguish serous carcinoma from endometrioid adenocarcinoma and ultimately to understand the molecular basis for the differences in biological behavior of these tumors.

Insulin-like growth factor-II mRNA-binding protein 3 (IMP3), also known as L523S or KOC (K-homologous domain-containing protein overexpressed in cancer), ${ }^{2,3}$ is a 580 residue protein containing four K homology domains and two other RNA recognition motifs. The IMP3 gene is located on chromosome 7p11.5. IMP3 is a member of the human IGF-II mRNA-binding protein family (IMPs) whose other members are IMP1 and IMP2. These proteins are capable of strong and specific binding with mRNA transcripts including those encoding insulin-like growth factor-II (IGF-II), cell adhesion molecules and others. IMPs have been implicated in various post-transcriptional processes, such as mRNA localization, ${ }^{4}$ turnover, ${ }^{5}$ and translational control. ${ }^{6}$

IMP3 is expressed in many cells of the developing fetus. In contrast, it is not expressed in most adult tissues except in the gonads. However, IMP3 is expressed in a number of cancers. A monoclonal antibody specific for IMP3 has been developed and used successfully with routine immunohistochemical methods to study overexpression of the protein in malignant pancreatic, renal, and uterine endocervical lesions. ${ }^{3,7,8}$ Expression of IMP3 in endometrial carcinomas has not been previously studied. The aims of this study were to evaluate the expression of IMP3 in endometrial carcinomas especially endometrioid adenocarcinoma and serous carcinoma subtypes as well as benign endometrium using immunohistochemical techniques, to assess the diagnostic utility of IMP3 in distinguishing endometrioid adenocarcinoma from serous carcinoma, to correlate IMP3 expression with clinicopathologic features of the disease, and to understand the possible role of IMP3 in the pathogenesis of endometrial adenocarcinoma.

\section{Materials and methods}

\section{Case Selection}

One hundred and sixty-seven cases of endometrial carcinoma with adequate archive tissue for immunohistochemical study were retrieved from the files of the Department of Pathology at UMass Memorial Medical Center accessioned between August 1999 and May 2006. These included 122 cases of endometrioid adenocarcinoma and 45 cases of serous carcinoma. All studied endometrioid adenocarcinoma specimens were from hysterectomy specimens. The specimens for serous carcinoma included 6 endometrial biopsies (EMB), 2 endometrial curettings (EMC), and 37 hysterectomies. The diagnosis of endometrioid adenocarcinoma or serous carcinoma was made in accordance with published diagnostic criteria. ${ }^{9}$ Tumor grade and stage were evaluated using the FIGO criteria. Twenty control samples of benign endometrium were obtained from 20 patients who underwent hysterectomy at UMass Memorial Medical Center in 2004 for benign uterine lesions including leiomyomas, adenomyosis, and endometrial polyps.

\section{Immunohistochemical Study}

Immunohistochemical stains were performed as described previously. ${ }^{3,7}$ Briefly, $5-\mu \mathrm{m}$ tissue sections were cut, deparaffinized, washed, and immersed in citrate buffer $(0.01 \mathrm{M}, \mathrm{pH} 6.0)$, and underwent microwave $(770 \mathrm{~W}, 14 \mathrm{~min})$ antigen retrieval. The slides were cooled, rinsed with PBS (three rinses, 5 min each), and stained on a DAKO Autostainer (Dako Corporation, Carpinteria, CA, USA) at room temperature. Endogenous peroxidase activity was blocked with $3 \%$ hydrogen peroxide, and the slides were rinsed and treated with a blocking protein (ChemMate; Ventana, Tucson, AZ, USA) to prevent nonspecific staining. The sections were then incubated with L523S, a mouse monoclonal antibody specific for IMP3 $(2 \mu \mathrm{g} / \mathrm{ml}$; Corixa Corporation, Seattle, WA, USA) for $45 \mathrm{~min}$, or with p53 mouse monoclonal antibodies (a cocktail of two clones: Ab2 at 1:300 dilution and Ab6 at 1:1000 dilution; Calbiochem, San Diego, CA, USA) for $30 \mathrm{~min}$. Following brief buffer washes, the sections were treated with a cocktail of biotinylated anti-rabbit IgG and antimouse IgG/IgM (ChemMate) for $30 \mathrm{~min}$, washed, and incubated with avidin-biotin-peroxidase complex (ChemMate) for $30 \mathrm{~min}$. The sections were rinsed, developed with diaminobenzidine (ChemMate) and hydrogen peroxide $(10 \mathrm{~min})$, rinsed with tap water, and counterstained with hematoxylin.

Representative sections of pancreatic carcinoma were used as positive controls for IMP3 expression, and sections of high-grade ovarian serous carcinoma were used as positive control for p53. Negative controls were performed by replacing the primary antibody with nonimmune IgG. The percentage of endometrioid adenocarcinoma, serous carcinoma, or normal endometrium that was positive for IMP3 or p53 was assessed. The intensity of the staining was evaluated as weak, intermediate, or strong.

\section{Statistical Study}

Independent sample $t$-tests was used to compare mean values of continuous variables in different groups. $P<0.05$ were considered to indicate significance.

\section{Results}

\section{Clinicopathologic Features}

One hundred and twenty-two patients with endometrioid adenocarcinoma ranged in age from 34 to 
89 years. The median age was 61 years. Thirty-four $(28 \%)$ cases had tumor cell nuclear grade 1,76 $(62 \%)$ cases grade 2 , and $12(10 \%)$ cases grade 3. Tumor architecture grade 1 was observed in 61 $(50 \%)$ cases, grade 2 in $53(43 \%)$ cases, and grade 3 in $8(7 \%)$ cases. FIGO stages of these 122 endometrioid adenocarcinomas were IA $(n=43)$, IB $(n=45)$, IIA $(n=5)$, IIB $(n=2)$, IIIA $(n=4)$, and IIIC $(n=6)$. Forty-five patients with serous carcinoma ranged in age from 35 to 85 years old with a median age of 68 years. Among these 37 cases of serous carcinoma with clinicopathologic stage assessed, 9 cases were diagnosed with stage IA disease, 6 stage IB, 4 stage IC, 1 stage IIA, 6 stage IIB, 4 stage IIIA, 6 stage IIIC, and 1 stage IVB disease. The age of the 20 control patients with hysterectomies performed for benign uterine lesions ranged from 34 to 58 years. Of these 20 cases, 6 had proliferative, 4 weakly proliferative, 8 secretory, and 2 inactive endometria. All 20 cases had leiomyomas. Additionally, 11 cases also had adenomyosis, and 3 had endometrial polyps.

\section{Immunohistochemical Findings}

\section{IMP3 expression}

All of the serous carcinoma cases showed cytoplasmic staining for IMP3 (Figure 1). As indicated in Table 1, 39 cases $(86 \%)$ showed positive staining for IMP3 in more than $50 \%$ of tumor cells, and 3 cases (7\%) had staining in $21-50$ or $6-20 \%$ of the tumor cells. Immunohistochemical intensity for IMP3 was found to be strong in 38 (84\%) and intermediate in $7(16 \%)$ serous carcinoma cases.

As shown in Table 1, 54 of $122(44 \%)$ endometrioid adenocarcinoma cases were negative for IMP3, and $68(56 \%)$ cases showed immunohistochemical expression of the protein. Among these 68 cases, 30 cases $(25 \%)$ demonstrated positive staining in $1-5 \%$ tumor cells, $20(16 \%)$ in 6-20\%, 10 (8\%) in $21-50 \%$, and $8(7 \%)$ in $>50 \%$ of the tumor cells. Strong IMP3-staining intensity was noted in 34 cases $(28 \%)$, intermediate in 26 cases $(21 \%)$, and weak in 8 cases $(7 \%)$.

The nuclear grade, architectural grade, FIGO stage, and patient's age at the diagnosis of endometrioid adenocarcinomas were compared between endometrioid adenocarcinomas with and without IMP3 expression. Endometrioid adenocarcinoma with IMP3 expression was defined as tumor with $>5 \%$ of the neoplastic cells showing positive staining for IMP3. Endometrioid adenocarcinomas with IMP3 expression had significantly higher nuclear and architectural grades than those without IMP3 expression ( $P=0.0000$ and $P=0.0002$, respectively). There was no FIGO stage or patient's age differences between these two groups $(P=0.5590$ and $P=0.8045$, respectively).

All 20 samples of morphologically benign endometrium evaluated were negative for IMP3. p53 expression

Positive immunohistochemical reaction for p53 in tumor cells was characterized by positive staining seen in the nucleus. As shown in Table 2, 35 out of $45(78 \%)$ serous carcinoma cases demonstrated strong p53 staining in more than $50 \%$ of the tumor cell nuclei. Thirty-two of $112(29 \%)$ endometrioid adenocarcinoma cases were negative for $\mathrm{p} 53$, and 80 $(71 \%)$ cases showed immunohistochemical expression of the protein. Among these 80 cases, 40 cases $(36 \%)$ demonstrated positive staining in $1-5 \%$ tumor cells, $23(20 \%)$ in $6-20 \%, 6(5 \%)$ in $21-$ $50 \%$, and $11(10 \%)$ in $>50 \%$ of the tumor cells. Strong p53-staining intensity was identified in 75 cases $(67 \%)$, intermediate in 4 cases $(3 \%)$, and weak in 1 case $(1 \%)$.

\section{Discussion}

Endometrial serous carcinoma is the most common aggressive form of endometrial malignancy. It has a strong predilection for extrauterine spread and poor clinical prognosis. The mechanism of its aggressive clinical behavior is unknown, although a recent focus has been done on a potential role for cellular adhesion molecules in this process. ${ }^{10}$ In this study, we have demonstrated that IMP3 is highly expressed in endometrial serous carcinoma as compared to endometrial endometrioid adenocarcinoma $(P<0.0001)$, and expression of IMP3 in the latter form, although low in frequency, correlates with higher tumor nuclear and architectural grades, suggesting that IMP3 may be of diagnostic value in differential diagnosis, a marker of tumor aggressive clinical behavior, and important in the pathogenesis of endometrial malignancy.

These results are of particular interest because IMP3 has been found to be a biomarker associated with the progression of other unrelated cancers. Thus its expression progressively increases with advancing stage in pancreatic cancers. ${ }^{3}$ Moreover, in renal cell carcinoma, IMP3 expression identifies the majority of cases that go on to metastasize and ultimately kill patients. ${ }^{7}$ Together these findings raise the possibility that IMP3 promotes aggressive behavior in tumors and might explain why most of the more highly aggressive serous carcinomas express this molecule as well as why it is present more frequently in high- vs low-grade endometrial carcinomas.

IMPs are primarily expressed during early embryogenesis and at mid-gestation in the mouse,${ }^{11}$ but not in most adult tissues. ${ }^{12,13}$ IMPs are considered to be oncofetal proteins that are frequently overexpressed in various cancers. IMPs play important roles in the binding, trafficking, and stabilization of the fetal subtype of IGF-II mRNA during embryogenesis. ${ }^{12,14}$ In a recent study to characterize the cellular functions of IMPs utilizing the RNA interference technique in cancer cells (HeLa), Vikesaa et al $1^{15}$ 

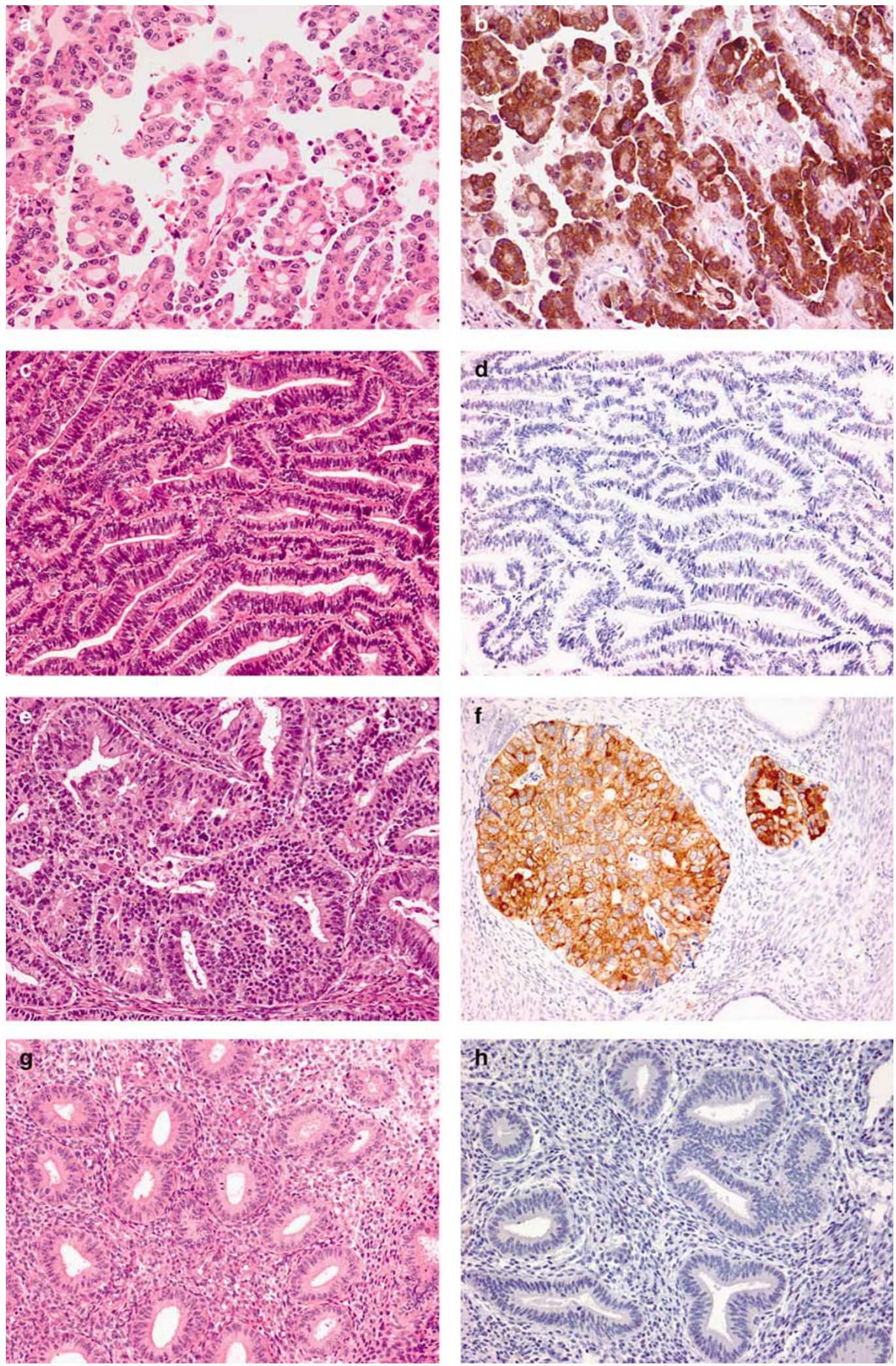

Figure 1 (a) Serous carcinoma of the endometrium (H\&E); (b) cytoplasmic expression of IMP3 in serous carcinoma of the endometrium; (c) International Federation of Gynecology and Obstetrics (FIGO) grade 1 endometrioid adenocarcinoma of the endometrium (H\&E); (d) negative immunohistochemical stain for IMP3 in FIGO grade 1 endometrioid adenocarcinoma of the endometrium; (e) FIGO grade 3 endometrioid adenocarcinoma of the endometrium (H\&E); (f) cytoplasmic expression of IMP3 in FIGO grade 3 endometrioid adenocarcinoma of the endometrium; (g) benign proliferative endometrium (H\&E); (h) negative immunohistochemical stain for IMP3 in benign proliferative endometrium. 
showed that IMPs are necessary for cell adhesion, cytoplasmic spreading, and invadopodia formation; implying that IMPs are involved in cellular adhesion and invasion during normal development and malignancy formation. Previous study demonstrated that antisense suppression of the IMP3 orthologue in xenopus embryos inhibited the migration of neural crest cells. ${ }^{16}$ Moreover, another study showed that transgenic overexpression of IMP3 in the pancreas resulted in increased proliferation and metaplasia of acinar cells. ${ }^{17}$ These findings might explain why IMP3 is associated with more aggressive cancers.

Further analysis of HeLa cells found that the loss of IMPs was associated with a coordinate downregulation of mRNAs encoding extracellular matrix and adhesion proteins, particularly, a $5.0 \mathrm{~kb} C D 44$ mRNA. ${ }^{15}$ CD44 protein is a member of Ig-superfamily cell adhesion molecules. It participates in intercellular adhesion, and promotes collagen IV degradation and tumor cell invasion by anchoring matrix metalloproteinases. ${ }^{18}$ These results suggest that $C D 44$ mRNA regulation may be involved in IMP-mediated tumor cell invasion and metastasis.

Several studies have shown that endometrial serous carcinoma has significantly less CD44 expression than endometrial endometrioid adenocarcinoma. ${ }^{19,20}$ The loss of CD44 expression has been correlated with a more aggressive course of endometrial carcinomas. ${ }^{21,22}$ CD44 expression is subject to both alternative splicing and post-translational modifications, ${ }^{23}$ so the selective regulation of the CD44 transcripts by IMPs including IMP3 provides an additional level of complexity to the control of CD44 expression. It has been hypothesized that CD44 transcripts may be divided into an IMPupregulating and an IMP-downregulating population. ${ }^{15}$ Our data imply that IMP-downregulating $C D 44$ transcripts may play an important role in the pathogenesis of uterine serous carcinoma. Downregulation of the adhesion protein CD44 and loss of invadopodia may collectively explain the reduced cell-cell adhesiveness in serous carcinoma and early dissemination of the carcinoma cells into the peritoneal cavity through the fallopian tubes.

Mutation of the p53 tumor suppressor gene accompanied by overexpression of the mutant p53 protein is one of the major genetic alterations in endometrial serous carcinoma. ${ }^{24}$ Therefore, p53 has been used as a biomarker for endometrial serous carcinoma. ${ }^{25}$ To correlate IMP3 and p53 expressions in endometrial carcinomas, immunohistochemical expression of p53 was evaluated in all 45 cases of serous carcinoma and 112 cases of endometrioid adenocarcinoma. Our data showed in Table 2 demonstrate that IMP3 may be another useful biomarker for endometrial serous carcinoma in difficult cases.

In summary, IMP3 is highly expressed in endometrial serous carcinoma as compared to endometrial endometrioid adenocarcinoma $(P<0.0001)$, and when expressed in endometrioid carcinomas it is 
more frequently found in tumors of higher grade. Therefore, in endometrial adenocarcinomas, as is in some other cancers, IMP3 expression is found in more aggressive disease.

\section{Acknowledgement}

We are grateful to the Corixa Corporation for providing the monoclonal antibody against IMP3, and to Mr Louis Savas for his technical support.

\section{References}

1 Creasman WT, Odicino F, Maisonneuve $\mathrm{P}$, et al. Carcinoma of the corpus uteri. J Epidemiol Biostat 2001;6:47-86.

2 Wang T, Fan L, Watanabe Y, et al. L523S, an RNAbinding protein as a potential therapeutic target for lung cancer. Br J Cancer 2003;88:887-894.

3 Yantiss RK, Woda BA, Fanger GR, et al. KOC (K homology domain containing protein overexpressed in cancer): a novel molecular marker that distinguishes between benign and malignant lesions of the pancreas. Am J Surg Pathol 2005;29:188-195.

4 Runge S, Nielsen FC, Nielsen J, et al. H19 RNA binds four molecules of insulin-like growth factor II mRNAbinding protein. J Biol Chem 2000;275:29562-29569.

5 Doyle GA, Betz NA, Leeds PF, et al. The c-myc coding region determinant-binding protein: a member of a family of $\mathrm{KH}$ domain RNA-binding proteins. Nucleic Acids Res 1998;26:5036-5044.

6 Liao $\mathrm{B}, \mathrm{Hu} \mathrm{Y}$, Herrick DJ, et al. The RNA-binding protein IMP-3 is a translational activator of insulin-like growth factor II leader-3 mRNA during proliferation of human K562 leukemia cells. J Biol Chem 2005;280: 18517-18524.

7 Jiang Z, Chu P, Woda BA, et al. Analysis of RNAbinding protein IMP3 to predict metastasis and prognosis of renal-cell carcinoma: a retrospective study. Lancet Oncol 2006;7:556-564.

$8 \mathrm{Li} \mathrm{C}$, Rock KL, Woda BA, et al. IMP3 is a novel biomarker for adenocarcinoma in situ of the uterine cervix: an immunohistochemical study in comparison with p16(INK4a) expression. Mod Pathol 2007;20:242-247.

9 Scully RE, Bonfiglio TA, Kurman RJ, et al. Histological Typing of Female Genital Tract Tumors. SpringerVerlag: New York, 1994, pp 13-31.

10 Leblanc M, Poncelet C, Soriano D, et al. Alteration of CD44 and cadherins expression: possible association with augmented aggressiveness and invasiveness of endometrial carcinoma. Virchows Arch 2001;438:78-85.

11 Hansen TV, Hammer NA, Nielsen J, et al. Dwarfism and impaired gut development in insulin-like growth factor II mRNA-binding protein 1-deficient mice. Mol Cell Biol 2004;24:4448-4464.

12 Nielsen J, Christiansen J, Lykke-Andersen J, et al. A family of insulin-like growth factor II mRNA-binding proteins represses translation in late development. Mol Cell Biol 1999;9:1262-1270.

13 Mueller-Pillasch F, Pohl B, Wilda M, et al. Expression of the highly conserved RNA binding protein KOC in embryogenesis. Mech Dev 1999;88:95-99.

14 Nielsen FC, Nielsen J, Christiansen J. A family of IGF-II mRNA binding proteins (IMP) involved in RNA trafficking. Scand J Clin Lab Invest 2001;234(Suppl): 93-99.

15 Vikesaa J, Hansen TVO, Jønson L, et al. RNA-binding IMPs promote cell adhesion and invadopodia formation. EMBO J 2006;25:1456-1468.

16 Yaniv K, Fainsod A, Kalcheim C, et al. The RNAbinding protein $\mathrm{Vg} 1 \mathrm{RBP}$ is required for cell migration during early neural development. Development 2003;130:5649-5661.

17 Wagner M, Kunsch S, Duerschmied D, et al. Transgenic overexpression of the oncofetal RNA binding protein KOC leads to remodeling of the exocrine pancreas. Gastroenterology 2003;124:1901-1914.

$18 \mathrm{Yu}$ Q, Stamenkovic I. Localization of matrix metalloproteinase 9 to the cell surface provides a mechanism for CD44-mediated tumor invasion. Genes Dev 1999; 13:35-48.

19 Soslow R, Shen P, Isacson C, et al. The CD44v6negative phenotype in high-grade uterine carcinomas correlates with serous histologic subtype. Mod Pathol 1998;11:194-199.

20 Hosford S, Elliott J, Ma ZW, et al. CD44 expression in papillary serous endometrial carcinoma. Int J Gynecol Cancer 2003;13:480-484.

21 Fujita N, Yaegeshi N, Ide Y, et al. Expression of CD44 in normal human vs tumor endometrial tissues: possible implication of reduced expression of CD44 in lymph-vascular space involvement of cancer cells. Cancer Res 1994;54:3922-3928.

22 Ayhan A, Tok E, Bildirici I, et al. Overexpression of CD44 variant 6 in human endometrial cancer and its prognostic significance. Gynecol Oncol 2001;80: 355-358.

23 Marhaba R, Zoller M. CD44 in cancer progression: adhesion, migration and growth regulation. J Mol Histol 2004;35:211-231.

24 Lax SF, Kendall B, Tashiro H, et al. The frequency of p53, K-ras mutations, and microsatellite instability differs in uterine endometrioid and serous carcinoma: evidence of distinct molecular genetic pathways. Cancer 2000;88:814-824.

25 Darvishian F, Hummer AJ, Thaler HT, et al. Serous endometrial cancers that mimic endometrioid adenocarcinomas: a Clinicopathologic and Immunohistochemical Study of a Group of Problematic Cases. Am J Surg Pathol 2004;28:1568-1578. 